\title{
Evaluation of Lowland Bamboo Propagation Techniques in West Hararghe Zone, Oromia Region, Ethiopia
}

\author{
Alemayehu Diriba*, Shimelis Dekeba, Wasihun Gizaw \\ Mechara Agricultural Research Centre, Mechara, Ethiopia \\ Email address: \\ alemayhudiriba@gmail.com (A. Diriba) \\ ${ }^{*}$ Corresponding author \\ To cite this article: \\ Alemayehu Diriba, Shimelis Dekeba, Wasihun Gizaw. Evaluation of Lowland Bamboo Propagation Techniques in West Hararghe Zone, \\ Oromia Region, Ethiopia. Journal of Energy and Natural Resources. Vol. 10, No. 3, 2021, pp. 65-70. doi: 10.11648/j.jenr.20211003.12
}

Received: March 26, 2021; Accepted: July 15, 2021; Published: July 23, 2021

\begin{abstract}
Bamboo has diverse utility ranging from construction to delicacy in Asia and Africa, and also several desirable qualities that make it a useful resource compared to many other plants. For example; it uses as food and feed preservative, construction, medicine purpose, natural conservation, charcoal and energy, arts and culture, aesthetic value, financial return and environment protection. Bamboo has additional unique characteristic that a fast growing plant than any other tree species and starts to give utility within three or four years of planting time in the exception of bearing fruits. To give fruit full seed takes a long time that after 50 years old. So to get its utilities has to be raised through various propagation methods. In this trial; propagations with culm cut, offsets cut and branch cut on Dendrocalmus membracius, Dendrocalmus hamlitonii and Oxytenanthera abyssinica bamboo species had been executed in RCBD design with three replications, respectively. So that in this experiment, among the methods of bamboo propagation technique; offset cutting (bamboo rhizome) followed by branch cut had been recommended based on consideration of giving adequate planting materials. The outcomes showed highly significant difference at $(p<0.001)$ level between and within treatments as well as interaction effect based on the given parameters. Regardless of this fact; the highest mean value discovered under Dendrocalmus hamlitonii bamboo species with culm cut planting followed by Dendrocalmus memebranceous with branch cut planting, while the least mean value was recorded under Oxytenanthera abyssinica with culm cut planting based on number of new emerging bamboo shoot parameter. Based on root collar diameter, Length of shoot and number of node parameters, the highest mean value recorded under Dendrocalmus hamlitonii and Dendrocalmus memebranceous with offset cut planting, while the least mean value was recorded under Dendrocalmus hamlitonii with branch cut planting, respectively (Table 2). Despite this fact, the recommend that with offset cut plating for Dendrocalmus hamlitonii and Dendrocalmus memebranceous of bamboo species followed by Oxytenanthera abyssinica. The Culm cut planting parts designated higher mean value for Dendrocalmus hamlitonii bamboo species only, while branch cut planting designated higher mean value for Dendrocalmus memebranceous bamboo species only (Table 2). Generally, the study was propagations of lowland bamboo species (Dendrocalmus membracius, Dendrocalmus hamlitonii and Oxytenanthera abyssinica) with culm cut, offsets cut and branch cut techniques under Mechara and for related agro ecology zones is trustworthy so, the research advocated these technologies with further economic and livelihood benefits for different stakeholders through expanding the plantation materials with full package.
\end{abstract}

Keywords: Exotic and Indigenous, Propagation Techniques, Lowland Bamboo Species, Unique Characteristics of Bamboo

\section{Introduction}

Bamboo belongs to the grass family and is mostly found in tropical, sub-tropical and in rare cases temperate zones. In terms of ecological habitat, it is classified into lowland and highland bamboo. Lowland bamboo grows naturally in tropical areas where temperature ranges from 20 to $35^{\circ} \mathrm{C}$ and rainfall is over $1500 \mathrm{~mm}$ per year. In Africa, indigenous bamboo grows mainly in the highlands, medium and lowland altitudes of Eastern and Central Africa. In nature, bamboo 
grows as pure stands or is mixed with other trees. Bamboo growing in forested catchments plays a vital role in the protection of soils and water sources [8].

Bamboo forms the bulk of elephant and wildlife feed and has diverse utility ranging from construction to delicacy in Asia and Africa, and it has also several desirable qualities that make it a useful resource compared to many other plants. It is tremendously valuable plant which has been used for hundreds of years with society. Long lasting accountability of bamboo with society is for its ability to restore degraded sites, short growing cycle, and ability to regenerate vegetativelly, tolerance to repeated harvesting, strong and lightweight material, and compatibility with other tree species [9].

Bamboo can be raised through various methods such as with seeds, wildings, culm cut, offsets cut, branch cut and tissue culture. However, culm cutting propagation technique is not suitable for solid or no hollow in the middle of the stem $[4,5,10]$. Indigenous and introduced species of bamboo do not readily produce seeds within a short period of time. This is taken a long time that after 50 years old to give fruit full seeds. Globally at present, the local demand for bamboo propaguller is greatest for ornamental purpose but that for its multipurpose utilities, bamboo is expected to get closer soon with put up their own plantations in very near future by many private landowners, and due these, its demined become double folded [2]. So that in order to compensate such imperative spots; its propagation methods had been identified with such kind of experiment [3].

In Ethiopia, there are a number of limitations at bamboo production and promotion thought out the regions those are agricultural expansion, lack of scientific knowledge on its management, propagation methods and utilization potential are the major bottlenecks for bamboo resources. But in this experiment, among the methods of bamboo propagation technique, offset cutting (bamboo rhizome) followed by branch cut had been recommended based on consideration of agro ecology zone that is as a faster providing of adequate planting material and higher regeneration percentage from each species [6].

Commonly in Ethiopia and particularly in Hararghe; as population size is increased, deforestation and land degradation problems have been gradually aggravating. So searching of alternative technology is mandatory. Therefore; important of the study is, to evaluate and document propagation methods of low land bamboos species with full package of economic and environmental values for all end users.

\section{Material and Methods}

\subsection{Description of the Study Areas}

The trial had been conducted at Mechara Agricultural Research Center (on station). The center is located at $431 \mathrm{Km}$ west of Addis Ababa. The altitude is 1650 m.a.s.l. The area experiences bimodal rainy season extending from March to October, but the effective rain is from May to September. The mean annual rainfall is about $1280 \mathrm{~mm}$ with a peak in July. Mean annual temperature is $20^{\circ} \mathrm{C}$, with mean minimum temperature of $13^{\circ} \mathrm{C}$ and maximum of $27^{\circ} \mathrm{C}$; and the soil of the area is dominantly reddish brown that is Nitosols (Mechara agricultural research center, meteorological station).

They are generally clay dominated and are characterized by low available phosphorous with a $\mathrm{pH}$ ranging from 5.3 to 6 in surface soils [1]. The vegetation cover of the area is of woodland and open wooded grassland types.

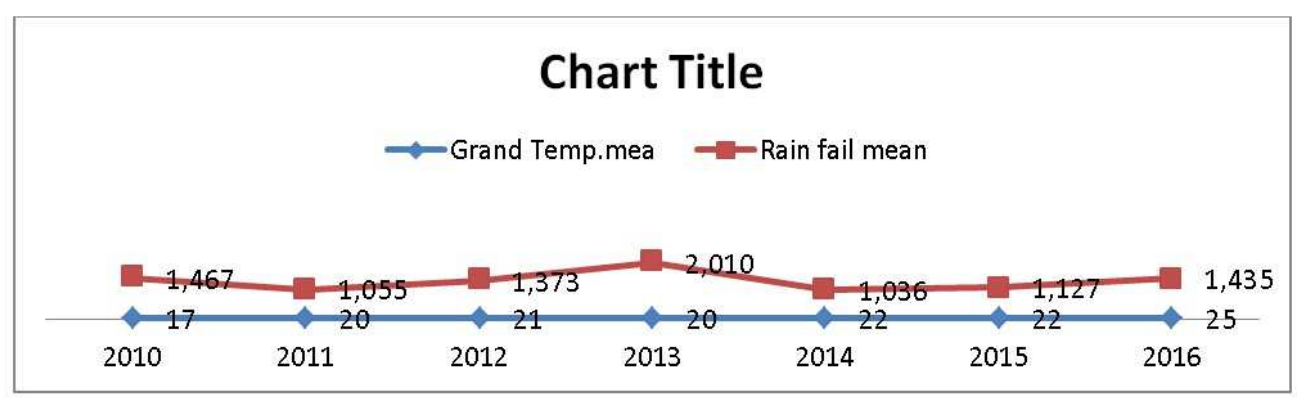

Figure 1. (2009-2016 G.C) Rainfall and temperature of Darolabu District 2016/2017.

\subsection{Planting Materials and Tending Operation}

The planting materials were taken from 2-3 years old of low land bamboos species namely (Dendrocalamus hamlitonii, Dendrocalamus memebranceous and Oxytenanthera abyssinica) and prepared based on manuals for tropical bamboos [7].

In this experiment, the propagating materials (treatments) were prepared by serving offset planting, culm cut planting and branch cut planting. The offset planting consists of a rhizome and a portion of the culm cut just including the $4^{\text {th }}$ or $5^{\text {th }}$ node from the base. Culm Cutting was planted from the near base of selected culm that had the branches of 2 or 3 internodes lengths with the cut positioned 2 to $3 \mathrm{~cm}$ below the basal node and water was poured into the cuttings through the holes. Branch cut planting method was performed with branches that have adventitious roots below the branch bases, "swollen" bases and 2 to 3 internodes length. The space between and within each planting materials were $2 \mathrm{~m}$ apart. On per plot, 3 propagation materials were taken from one bamboo species and similarly for others. A total of 27 planting materials from three bamboo species at 
total areas of $5.5 \mathrm{~m} * 20.5 \mathrm{~m}\left(112.75 \mathrm{~m}^{2}\right)$ were planted in experimental site. The planting technique of all parts was inclined horizontally with $45^{\circ}$ in the prepared pit. The pit was prepared with $50 \mathrm{~cm}$ deep and $50 \mathrm{~cm}$ wide.
The tools using were a sharp axe, saw, sickle, meter and dagger to separate from parent rhizome and culms as well as to dig and measure.

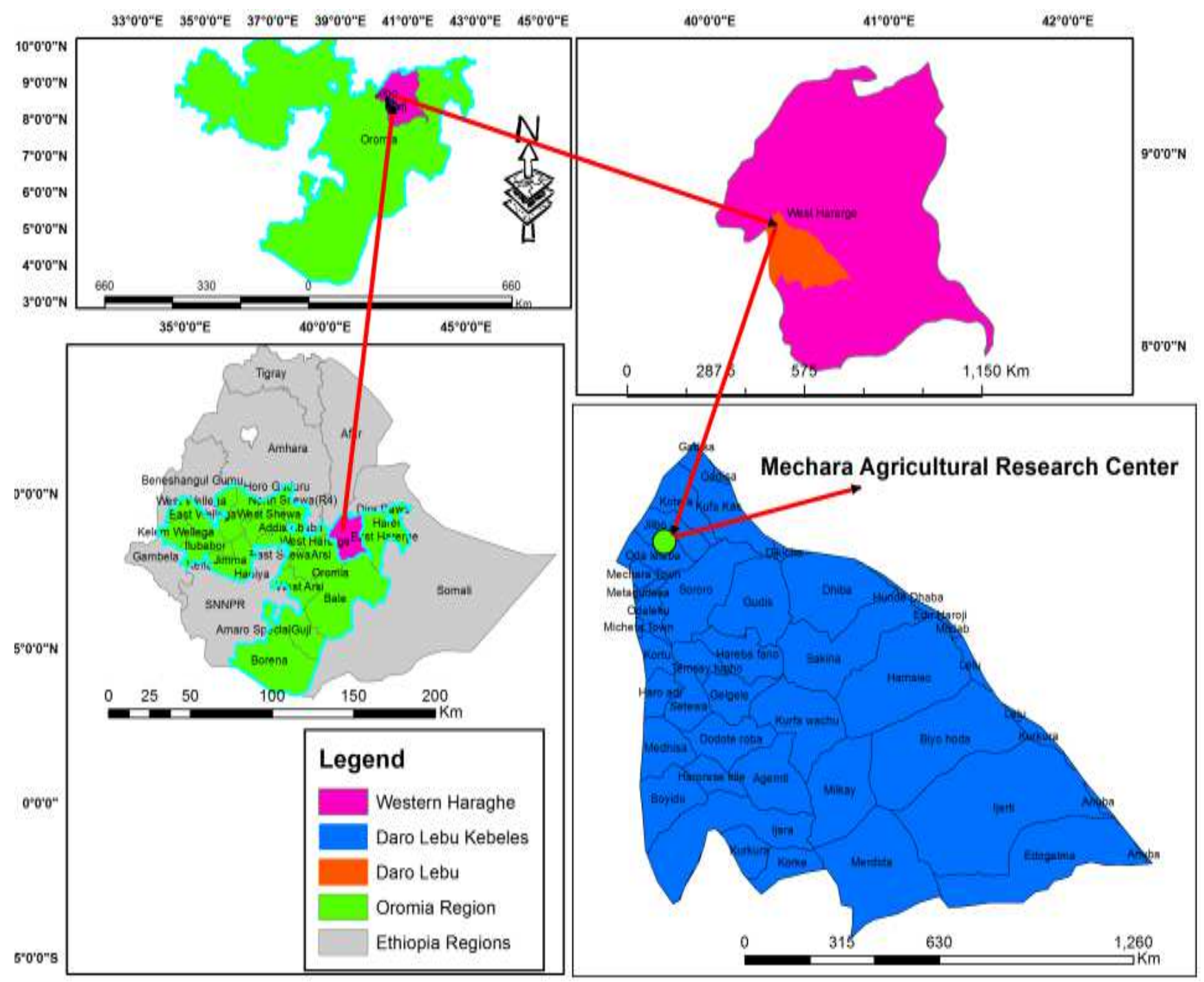

Figure 2. Description of Daro labu Woreda.

\subsection{Treatments, Experimental Design, Data Collection and Data Analysis}

Each bamboo species (Dendrocalamus hamlitonii, Dendrocalamus memebranceous and Oxytenanthera abyssinica) with its offset planting, culm cut planting and branch cut planting parts were used as treatments. The experimental designed had been implemented by Randomized Complete Block Design (RCBD) factorial with three replications. The collected data were emerging time taken of planting materials, regeneration percentage, number of new emerging bamboo shoots, root collar diameter, length of shoots and number of nodes. The collected data was examined with SAS, version 9.0 and interpreted significance difference between treatments' mean value based on a given parameters.
Table 1. Treatments of a given experiment.

\begin{tabular}{lll}
\hline \multirow{2}{*}{ No. } & Treatments & \\
\cline { 2 - 3 } & Bamboo species & Grafting parts of Bamboos \\
\hline \multirow{3}{*}{1} & Dendrocalamus hamlitonii & $\begin{array}{l}\text { offset planting/secure } \\
\text { culm cut planting } \\
\text { branch cut planting parts }\end{array}$ \\
& offset planting/secure \\
& Dendrocalamus & culm cut planting \\
& memebranceous & branch cut planting parts \\
& & offset planting/secure \\
& Oxytenanthera abyssinica & culm cut planting \\
& branch cut planting parts \\
\hline
\end{tabular}




\section{Result and Discussions}

\subsection{Emerging Time Taken of Planting Materials}

The present study indicated that the interaction effect and the mean value of the given parameters that emerging time taken of planting materials were revealed highly significant difference at $(\mathrm{p}<0.001)$ level within the planted parts and species of bamboo. The shortest value of emerging time taken of planting materials were seen from Dendrocalamus hamlitonii offset planting/secure and Oxytenanthera abyssinica culm cut planting, while the highest value were seen from Oxytenanthera abyssinica Offset cut and Dendrocalamus hamlitonii culm cut planting (Table 2). In this parameter, from the selected cutting part of bamboo species, the shortest time taken of emerging has to be taken as the best one is due to its shortest time taken to be emerged within a shortest duration than the others of a given treatments' materials (Table 2).

\subsection{Regeneration Percentage of Bamboo Materials}

The mean value and interaction effect of the given parameters that on regeneration percentage of bamboo materials showed highly significant difference at the level of $(p<0.001)$ point within the planted parts and species of bamboo (Table 2). The highest mean value of a given results were recorded from Dendrocalamus hamlitonii species and offset planting parts followed by Dendrocalamus hamlitonii species with culm planting and Oxytenanthera abyssinica species with offset planting, while the lowest mean values were recorded from Dendrocalamus hamlitonii species with branch cut planting and Oxytenanthera abyssinica species with culm cut planting respectively (Table 2).

The highest mean value of $a$ given treatments' parameter was greater than that of the lowest mean value is 66.7 percent (Table 2). This is difference might be due to environmental factors as well as bamboo species and part of cutting performances.

\subsection{Number of New Emerging Shoots}

The mean value of the given treatments' parameters showed highly significant difference at the level of $(p<0.001)$ point within the planted parts and species of bamboo. The capacity of lateral buds forming new rhizome and shoot is might be closely related to rhizome species, age, vigor, and nutrient storage. Based on the analysis results of the given data of Dendrocalamus hamlitonii species with culm cut planting revealed a highest mean value on the number of new emerging shoots followed by Dendrocalamus Membracius species with brunch cut planting, while the lowest mean value was showed from Oxytenanthera abyssinica species with culm cut planting part (Table 2).

The mean value of Dendrocalamus hamlitonii species with culm cut planting part on the given parameter was greater than that of Oxytenanthera abyssinica species with culm cut planting part by $84.3 \%$ (Table 2). This is might be due to high capability of producing new emerging shoots of the species when compare to the other planting part and specie of bamboo. This result is similar with the report from Bako Agricultural Research Center, 2016; which shows higher shoot emerging for Dendrocalamus hamlitonii species. Whereas, Oxytenanthera abyssinica species revealed a low performance in emerging a new number of shoots during the pursue periods (Table 1). On the opposite side of this result is revealed that the sprout of new shoots between the propagation part was similar except dry and wet season, reported by [11].

\subsection{Root Collar Diameters}

The mean value and interaction effect of the given parameter that on root collar diameter showed highly significant difference at the level of $(p<0.001)$ point within planted parts and species of bamboo. The highest mean was observed from Dendrocalamus memebranceous species with offset cut planting followed by Dendrocalamus membracius species with branch cut planting, while the least mean was recorded from Oxytenanthera abyssinica with culm cut planting, respectively (Table 2). The highest mean value of Dendrocalamus memebranceous species with offset cut planting is greater than that of the least mean value of Oxytenanthera abyssinica with culm cut planting by $81.3 \%$. This might be based on the growth performance and adaptability of the species (Table 2). This result is in line with the report of World Scientific journals that is the mean value of root collar diameter (RCD) a highly significant difference on the number highland bamboos species' shoots at the beginning of the first six months [12].

\subsection{Length of Emerging Shoot}

The mean value and interaction effect of the given parameters that on length of emerging shoot showed highly significant difference at the level of $(\mathrm{p}<0.001)$ point within planted parts and species of bamboo. The highest mean value $\mathrm{s}$ of a given treatments' parameter observed from Dendrocalamus hamlitonii species and Dendrocalamus memebranceous species both with offset cut planting, while the least mean values were recorded from Dendrocalamus hamlitonii species with branch cut planting followed by Oxytenanthera abyssinica species with culm cut planting, respectively.

The highest mean value of a given treatments' parameter greater than that of the least mean value is observed by $40 \%$ (Table 2). The result agreed with the report from Pawe by Yared k, 2013 (unpublished) which shows higher Culm height for Dendrocalamus hamlitonii species. On the other hands, these result disagreed with the report from Bako Agricultural Research Center, 2016; on Oxytenanthera abyssinica value. The full length of emerging shoot is varied among the species and part of cuttings; this disparity might be due species vary on its adaptability and performance conditions. 


\subsection{Number of Node}

The mean value of the given parameter that on number of node showed highly significant difference at the level of $(p<0.001)$ point within planted parts and species of bamboo. The highest mean value was observed from Dendrocalamus memebranceous species with offset cut planting that followed by Dendrocalamus hamlitonii species with offset cut planting, while the least mean values were recorded from Dendrocalamus hamlitonii and Oxytenanthera abyssinica species with branch cut and culm cut planting, respectively (Table 2). Based on the given parameter, Dendrocalamus memebranceous species with offset cut planting mean greater than that of Dendrocalamus hamlitonii and Oxytenanthera abyssinica species with branch cut and culm cut planting is by $86.5 \%$, respectively (Table 1 ).

Generally, Dendrocalamus hamlitonii and Dendrocalamus memebranceous show higher number of internodes as compare to others which is similar with the report of Yared K, 2013 (unpublished) which show higher number of internodes for Dendrocalamus hamlitonii. On the other hands, these results showed disagree with the report from Regassa Terefe et al., 2016 at the numbed of internodes Oxytenanthera abyssinica species.

Table 2. ANOV table of treatments' value based on the given parameters.

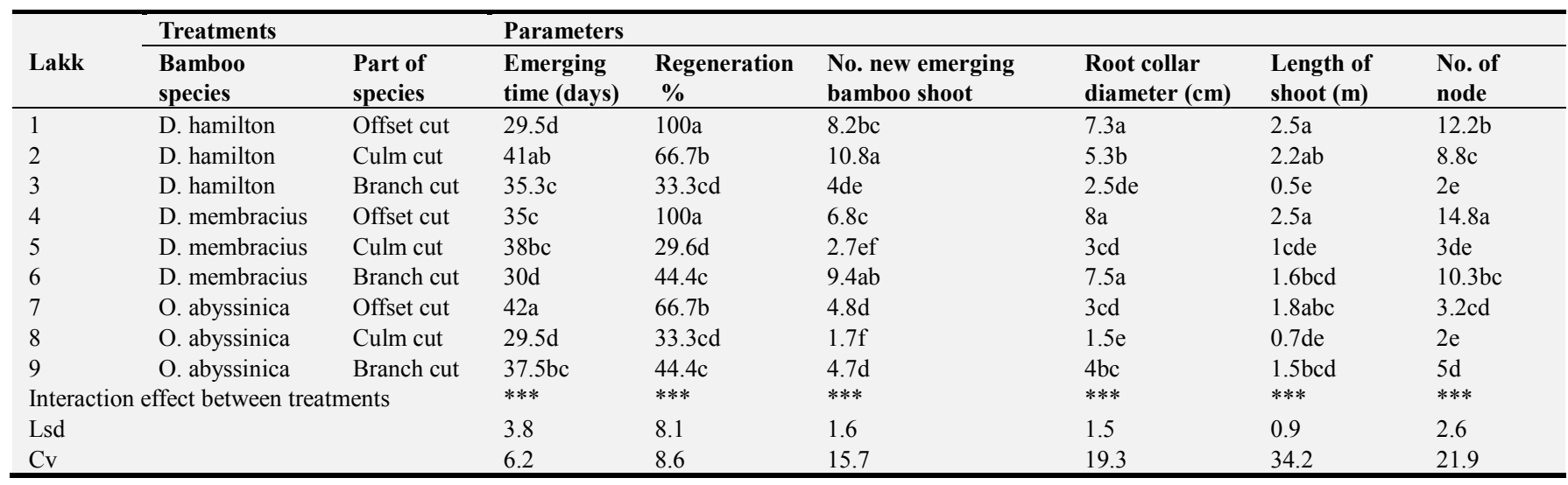

*(Vertically mean values with the same letter are not significantly different; $\mathrm{No}-$ Number, $\mathrm{cm}=$ centimeter, $\mathrm{m}=$ meter, $\mathrm{D}=$ Dendrocalamus and $\mathrm{O}=\mathrm{Oxytenanthera}$ )

\section{Conclusion and Recommendations}

Adaptation trial of lowland bamboo species had been took placed under Mechara condition and likewise for similar agro ecology zones as to be more affordable, available and acceptable for farmers rather than some miner variation on growth performances. Therefore, Mechara Agricultural Research Center believed that to disseminate the more adaptable bamboo species with recommended propagation techniques for all an end users with full packages. Because, bamboo species naturally long last to give seed. So propagation techniques are mandatory. Based on the previous research gap.

Therefore, as a conclusion that with regardless of this fact, the highest mean value of Oxytenanthera abyssinica specie was showed with offset cut plating, while the least mean value was recorded from Dendrocalmus hamlitonii and Oxytenanthera abyssinica with offset cut plating and culm cut planting based on emerging time taken parameter (Table 2).

Based on regeneration percentage parameter the highest mean value found under Dendrocalmus hamlitonii and Oxytenanthera abyssinica with offset cut plating, while the least mean value was recorded from Dendrocalmus memebranceous with culm cut planting, respectively (Table 2).

On the other necessary parameter, the highest mean value discovered under Dendrocalmus hamlitonii bamboo species with culm cut planting followed by Dendrocalmus memebranceous with branch cut planting, while the least mean value was recorded under Oxytenanthera abyssinica with culm cut planting based on number of new emerging bamboo shoot parameter. Based on root collar diameter, Length of shoot and number of node parameters, the highest mean value recorded under Dendrocalmus hamlitonii and Dendrocalmus memebranceous with offset cut planting, while the least mean value was recorded under Dendrocalmus hamlitonii with branch cut planting, respectively (Table 2).

Generally, the best recommendation had been given based on a given parameters' mean value. So the highest mean value indicated with offset cut plating for Dendrocalmus hamlitonii and Dendrocalmus memebranceous of bamboo species followed by Oxytenanthera abyssinica. The Culm cut planting parts designated higher mean value for Dendrocalmus hamlitonii bamboo species only, while branch cut planting designated higher mean value for Dendrocalmus memebranceous bamboo species only (Table 2).

Finally these trail indicate that the further research study need with this operation on other bamboo species. The other point has to be considered is; promotion and dissemination of the selected bamboo species with the recommended propagation methods. The future research track must be intended on provenance study and adaptation of highland bamboo, and its propagation techniques. 


\section{Acknowledgements}

The authors express their gratitude to Mechara Agriculture Research Center for activates work-done, and Oromia Agricultural Research Institution for its financial support.

\section{References}

[1] Dwivedi, A. P. 1993. Forests: The non-wood resources. International Book distributors, Dehra Dun, India. 352 pp. forest management.

[2] Fu Maoyi., 2005. Bamboo resources and utilization in China.

[3] John, C. K. and R. S. Nadgauda. 2002. Bamboo flowering and Famine. Current Science, 82 (3): 261-262.

[4] Kigomo, B. N. (1995). Guidelines for Establishment and Managing Plantations of Bamboo in Kenya. Occasional Management Paper No. 1. Kenya Forestry Research Institute, Muguga, Kenya.

[5] Kigomo, B. N. (2007). Guidelines for Growing Bamboo. Kenya Forestry Research Institute. Guidelines Series No. 4. 58 pp.
[6] Mekonnen, Z., Worku, A., Yohannes, T., Alebachew, M., and Kassa, H. (2014) Bamboo.

[7] Njuguna and Kigomo, 2008. Tropical bamboos history.

[8] Okamura, H. (1986). The Horticultural Bamboo Species in Japan.

[9] Ong, C. (2004). Giant Bamboo: A Grass Becomes a Multipurpose Tree.

[10] Ray, S. S., and Ali, M. N. (2017). Factors affecting macro propagation of bamboo with Research and Applications, 12, 511-524. dx.doi.org/10.1016/j. Resources in Ethiopia: Their value chain and contribution to livelihoods. Ethnobotany: Science, 47 (1), 17: DOI 10.1186/s40490-017-0097.

[11] Senyanobe, Journal, 2013). Response of vegetative propagation of Bambusa spps under special reference to culm cuttings: a review update. New Zealand Journal of Forestry.

[12] World Scientific News, 2017. Highland bamboos species' characteristics. 